\title{
Catecholamine Synthesis is Mediated by Tyrosinase in the Absence of Tyrosine Hydroxylase
}

\author{
Maribel Rios, ${ }^{1}$ Beth Habecker, ${ }^{2}$ Toshikuni Sasaoka, ${ }^{3}$ Graeme Eisenhofer, ${ }^{2}$ Hua Tian, ${ }^{2}$ Story Landis, ${ }^{2}$ \\ Dona Chikaraishi, ${ }^{4}$ and Suzanne Roffler-Tarlov ${ }^{1}$ \\ ${ }^{1}$ Departments of Neuroscience and Anatomy and Cell Biology, Tufts University Medical School, Boston, Massachusetts \\ 02111, ${ }^{2}$ National Institute of Neurological Diseases and Stroke, National Institutes of Health, Bethesda, Maryland 20892, \\ ${ }^{3}$ National Institute of Neuroscience, National Center for Neurology and Psychiatry, Tokyo 187, Japan, and ${ }^{4}$ Department of \\ Neurobiology, Duke University Medical Center, Durham, North Carolina 27710
}

Catecholamine neurotransmitters are synthesized by hydroxylation of tyrosine to L-dihydroxyphenylalanine (L-Dopa) by tyrosine hydroxylase $(\mathrm{TH})$. The elimination of $\mathrm{TH}$ in both pigmented and albino mice described here, like pigmented TH-null mice reported previously (Kobayashi et al., 1995; Zhou et al., 1995), demonstrates the unequivocal requirement for catecholamines during embryonic development. Although the lack of TH is fatal, TH-null embryos can be rescued by administration of catecholamine precursors to pregnant dams. Once born, TH-null pups can survive without further treatment until weaning. Given the relatively rapid half-life of catecholamines, we expected to find none in postnatal TH-null pups. Despite the fact that the TH-null pups lack TH and have not been supplemented with catecholamine precursers, catecholamines are readily detected in our pigmented line of $\mathrm{TH}$-null mice by glyoxylic acid-induced histofluorescence at postnatal day 7 (P7) and P15 and quantitatively at P15 in sympathetically inner- vated peripheral organs, in sympathetic ganglia, in adrenal glands, and in brains. Between 2 and $22 \%$ of wild-type catecholamine concentrations are found in these tissues in mutant pigmented mice. To ascertain the source of the catecholamine, we examined postnatal TH-null albino mice that lack tyrosinase, another enzyme that converts tyrosine to L-Dopa but does so during melanin synthesis. In contrast to the pigmented TH-null mice, catecholamine histofluorescence is undetectable in postnatal albino mutants, and the catecholamine content of TH-null pups lacking tyrosinase is $18 \%$ or less than that of TH-null mice with tyrosinase. Thus, these extraordinary circumstances reveal that tyrosinase serves as an alternative pathway to supply catecholamines.

Key words: catecholamines; tyrosine hydroxylase-null mutation; tyrosinase; tyrosine hydroxylase; tyrosinase and catecholamine synthesis; catecholamines in development; catecholamine synthesis
The catecholamines dopamine, norepinephrine, and epinephrine are synthesized from dietary tyrosine in selected central and peripheral neurons and in the adrenal medulla by the sequential action of enzymes in a synthetic pathway first postulated by Blaschko (1939) and finally fully demonstrated by Nagatsu et al. (1964) with the isolation of tyrosine hydroxylase (TH). Tyrosine hydroxylase, the first and rate-limiting enzyme in catecholamine synthesis, catalyzes the conversion of tyrosine to L-dihydroxyphenylalanine (L-Dopa), a substrate for Dopa decarboxylase (DDC) (aromatic amino acid decarboxylase), which converts L-Dopa to dopamine. DDC is ubiquitously distributed but is particularly abundant in the kidney and in catecholamineproducing cells. Dopamine serves as a neurotransmitter in cell groups in the brain and in a few peripheral groups. In noradrenergic neurons in the sympathetic nervous system, in the brainstem, and in adrenal chromaffin cells, dopamine is converted to norepinephrine by dopamine $\beta$ hydroxylase $(\mathrm{D} \beta \mathrm{H})$, whose expression is primarily restricted to these cells. Norepinephrine is

\footnotetext{
Received Sept. 30, 1998; revised Jan. 25, 1999; accepted Feb. 18, 1999.

This work was supported by National Institutes of Health Grant NS 31673 and by National Institute of Neurological Diseases and Stroke (NINDS) intramural program. We thank Dr. Irwin Kopin (NINDS, National Institutes of Health), whose suggestion put us on the trail of tyrosinase as a source of Dopa. We thank Dr. S. Tonegawa for his generous help in making the TH-null mutants.

Correspondence should be addressed to Suzanne Roffler-Tarlov, 136 Harrison Avenue, Boston, MA 02111.

Copyright (C) 1999 Society for Neuroscience $\quad 0270-6474 / 99 / 193519-08 \$ 05.00 / 0$
}

converted to epinephrine by phenylethanolamine- $N$-methyl transferase present in adrenergic cells in the adrenal medulla and in a few neuronal groups in the lower brainstem.

The synthetic pathway originally proposed (Blaschko, 1939) is the predominant route for catecholamine synthesis. Although alternative pathways may exist in mammals, none has ever been demonstrated to supply neurotransmitters. For example, tyrosinase, a key enzyme in melanin biosynthesis, also converts tyrosine to L-Dopa (Sanchez-Ferrer et al., 1995). Tyrosinase is abundant in melanocytes but, because melanocytes lack the other catecholamine synthetic enzymes, they do not synthesize catecholamines. Although L-Dopa produced in melanocytes could in principle enter the blood stream and subsequently be converted to catecholamines in neurons and chromaffin cells, there is no evidence for such a scenario in normal animals. We have examined two types of mutant mice, one that lacks $\mathrm{TH}$ and a second that lacks both $\mathrm{TH}$ and tyrosinase. We report here that, in the absence of $\mathrm{TH}$, tyrosinase contributes to catecholamine synthesis in the brain and peripheral cells that normally synthesize catecholamines via TH. These results raise the possibility that L-Dopa synthesized by tyrosinase is used for catecholamine synthesis under normal circumstances, as well.

\section{MATERIALS AND METHODS}

Construction of the targeting vector. TH was cloned from a $129 \mathrm{SV}$ mouse genomic library using a rat TH cDNA probe. As a targeting strategy, sequence within exons 6,7 , and 8 of the mouse TH gene that encodes for 
a.

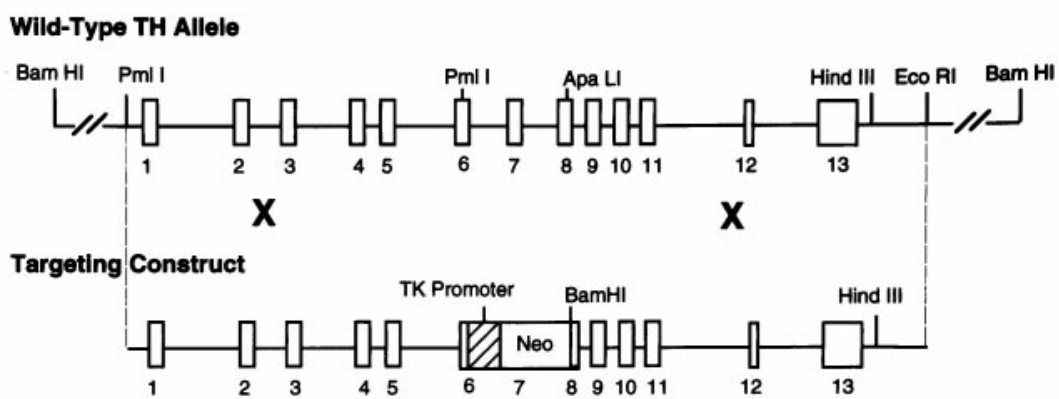

Figure 1. $a$, Disruption of the TH gene. Sequence between exons 6 and 8 of the mouse TH gene was replaced by a TK-Neo cassette without a polyadenylation signal. Vertical rectangles represent exons, and the short shaded horizontal bar represents the location of the 5' DNA probe. $b$, Southern blot analysis of an E12.5 litter obtained from a heterozygous cross. DNA extracted from E12.5 fetuses was digested with BamHI and HindIII and hybridized to the $5^{\prime}$ probe. The 13 and $10 \mathrm{~kb}$ bands represent the wild-type and targeted alleles, respectively. $c$, Western blot containing protein extracted from heads of E14.5 wild-type (lane 2), heterozygous (lane 3), and TH-null (lane 4) fetuses. Protein samples from $\mathrm{J} 1$ embryonic stem cells were a negative control (lane 1). Reduced content of TH protein was observed in the heterozygous fetuses compared with wild types. A low level of mutant TH was detected in extracts from the TH-null fetus.

TH Targeted Allele

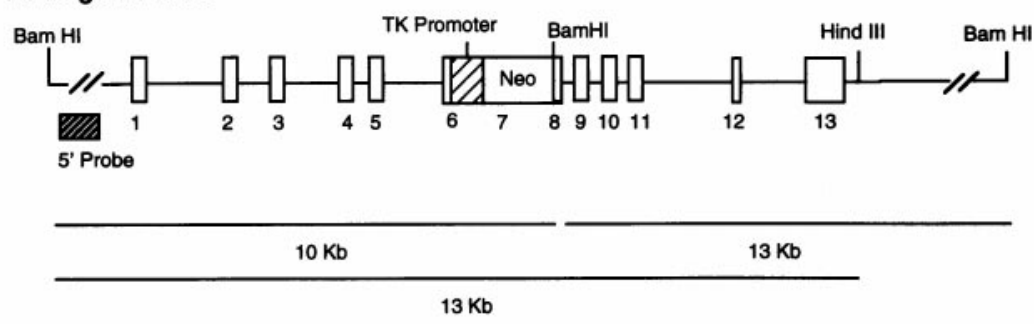

b.

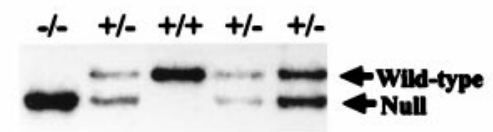

c.

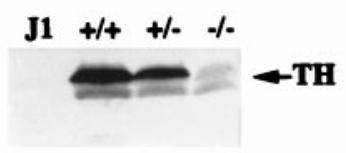

part of the catalytic domain was replaced by a thymidine kinaseneomycin (TK-Neo) cassette without a polyadenylation signal. This polyadenylation trap targeting vector was constructed in pMC-1 Neo. The TK-Neo cassette was inserted within mouse TH exons 6 and 8 using the PmlI site in exon 6 and the ApaLI site in exon 8. The $5^{\prime}$ and $3^{\prime}$ ends of the targeting vector contained 5.5 and $4.4 \mathrm{~kb}$ of homologous sequence with the TH wild-type allele, respectively.

Embryonic stem cell transfection and selection. The D3 embryonic stem (ES) cells from the $129 \mathrm{SV}$ strain provided by Dr. S. Tonegawa (Center for Memory and Learning, Massachusetts Institute of Technology, Cambridge, MA) were transfected by electroporation with $50 \mu \mathrm{g}$ of linearized TH targeting vector DNA. After selection in G418, neomycin-resistant clones were analyzed for the presence of a targeted allele using Southern blot analysis.

ES cells from homologous recombinant clones were injected into C57BL/6J blastocysts, which were transferred into the uteri of B6CBAF1 pseudopregnant females. Chimaeras were crossed with $\mathrm{C} 57 \mathrm{BL} / 6 \mathrm{~J}$ mice, and heterozygous carriers were crossed to produce pigmented TH-null animals. In addition to the pigmented TH-null mice, we created a second line of mutant mice that also lacked tyrosinase. To generate these mice, heterozygous carriers were backcrossed onto albino [homozygous tyrosinase (C locus)-deficient] ICR mice for three generations, and progeny from crosses between F3 heterozygous carriers and their progeny were used here as tyrosinase-deficient.

Southern blot analysis for detection of targeted TH allele. DNA extracted from individual neomycin-resistant clones was digested with Bam HI and hybridized to a 5' probe (Fig. 1). The hybridization yielded a $23 \mathrm{~kb}$ band arising from the TH wild-type allele or a $10 \mathrm{~kb}$ band from the TH targeted allele because of the introduction of a third BamHI site in the TK-Neo cassette.

For the screening of animals, tail fragments were cut from deeply anesthetized mice and digested in an SDS-Proteinase K solution. Extracted tail DNA was digested with BamHI and HindIII and hybridized to the $5^{\prime}$ probe, generating a 13 and a $10 \mathrm{~kb}$ band from a TH wild-type and a $\mathrm{TH}$ targeted allele, respectively.
Western blots. To examine TH protein content, protein was extracted from the heads of embryonic day 14.5 (E14.5) wild-type, heterozygous, and homozygous mutant fetuses and J1 embryonic stem cells (negative control). Samples were separated on a $12 \%$ acrylamide gel, transferred to blots, and probed with monoclonal and polyclonal antibodies to $\mathrm{TH}$ (DiaSorin Inc., Stillwater, MN and Sigma, St. Louis, MO) [polyclonal antibody obtained from Dr. William Tank (Department of Pharmacology, University of Rochester Medical Center, Rochester, NY)] using the ECL detection system (Amersham, Arlington Heights, IL).

Rescue of TH-null embryos with administration of L-Dopa or D, L-threo3,4-dihydroxyphenyl serine. Pregnant heterozygous females mated to heterozygous males were treated with L-Dopa or D,L-threo-3,4dihydroxyphenyl serine (Dops) (Research Biochemicals, Natick, MA) from E8.5 and until parturition. L-Dopa and Dops were administered in the drinking water at a final concentration of 1.0 and $0.5 \mathrm{mg} / \mathrm{ml}$, respectively. Water containing the drugs was shielded from light, changed daily, and included $0.25 \%$ ascorbic acid to reduce oxidation. Administration of the catecholamine precursors was discontinued at the time of birth.

Glyoxylic acid-induced catecholamine histofluorescence. Catecholaminecontaining cells and fibers were identified using the glyoxylic acid method (De la Torre, 1980). Ten micrometer cryostat sections of fresh frozen tissues were dipped in a solution containing $1 \%$ glyoxylic acid, $0.2 \mathrm{M}$ potassium phosphate, and $0.2 \mathrm{M}$ sucrose, $\mathrm{pH}$ 7.4. The sections were dried, heated to $95^{\circ} \mathrm{C}$ for $2.5 \mathrm{~min}$, and coverslipped in mineral oil.

Quantitative detection of catecholamines. Tissues were homogenized in cold $0.4 \mathrm{M}$ perchloric acid. Homogenates were centrifuged at $4^{\circ} \mathrm{C}$, and supernatants were stored at $-80^{\circ} \mathrm{C}$. Catechols were adsorbed onto alumina, eluted, separated by HPLC, and quantified by electrochemical detection as described previously (Eisenhofer et al., 1986). Differences among pigmented and albino groups and TH-null, heterozygote, and wild-type groups were analyzed by two-way ANOVA with post hoc tests of significance at the $p<0.05$ level determined using Sheffé's method. Differences among pigmented and albino TH-null mice, as percentages of respective wild-type values, were assessed by Student's $t$ test. All data were logarithmically transformed before statistical analysis. 


\section{RESULTS}

\section{Homologous recombination at the TH locus}

Mouse TH is encoded by a single gene with 13 exons from which a single transcript is derived (Ichikawa et al., 1991). A stretch of 268 bases from exon 6 to 8 that encodes part of the essential catalytic domain (Ribeiro et al., 1993) was replaced by a TK-NeO cassette. The targeting vector (Fig. 1a) transfected into D3 ES cells generated three homologous recombinants identified by Southern blot analysis. Two were injected into C57BL/6J blastocysts to generate seven chimeras, five of which produced heterozygous carriers when crossed with normal C57BL/6J mice. All heterozygous mice appeared to be normal and were intercrossed to generate litters that contained TH-null, heterozygous, and wild-type progeny with the expected Mendelian frequency when midgestation embryos were genotyped (Fig. 1b). Similar results were obtained with animals obtained from two independent ES cell homologous recombinant clones.

\section{TH expression}

Western blot analysis of protein extracted from the heads of wild-type, heterozygous, and TH-null E14.5 fetuses showed reduced content of TH protein in heterozygous animals compared with wild-type fetuses (Fig. 1c). A small amount of mutant TH protein close to the size of authentic TH $(60 \mathrm{kDa})$ was derived from the $\mathrm{TH}$ targeted allele (Fig. 1c). This band was detected with three antibodies that recognize different $\mathrm{TH}$ epitopes. The mutant $\mathrm{TH}$ protein could not be detected immunohistochemically in fetal adrenals or brains or in postnatal brain tissue (data not shown), suggesting that the mutant $\mathrm{TH}$ protein does not survive fixation. The lower band in the Western blot appears to be a degradation product of the major $\mathrm{TH}$ band.

\section{The TH-null mutants}

No pigmented TH-null mice survived to term of 510 newborns from heterozygous crosses. TH-null survival rates from other lines of pigmented TH-null mice range from 0 to $8 \%$ (Kobayashi et al., 1995; Zhou et al., 1995). Our analysis of staged litters suggests that the pigmented TH-null embryos begin to die at E9.5 and continue to do so throughout the midgestational period so that at E14.5 only $32 \%$ of TH-null embryos remain alive. Many of our TH-null fetuses fail to develop a normal heart, as reported by Zhou et al. (1995). Of four live TH-null fetuses examined at E12.5 and E13.5, three showed dilated atria, reduced cell density in the ventricles, and thinning of the atrial wall (data not shown). Eight of 19 TH-null fetuses had blood congestion in the heart and major blood vessels. Pools of blood were also present in the liver and lungs of some, but not all, mutants at E11.5, E12.5, and E13.5. No other obvious morphological abnormalities were observed. Although we have not yet analyzed our line of tyrosinasedeficient TH-null mice as thoroughly as the pigmented line, the lack of TH is also lethal to albino embryos. Thus, the pigmented and albino TH-null mutants reported here and other pigmented TH null mice described previously (Kobayashi et al., 1995; Zhou et al., 1995) are dependent on catecholamines for survival during embryonic life.

\section{Absence of glyoxylic acid-induced histofluorescence in pigmented null embryos}

To determine whether there are detectable catecholamines in the absence of wild-type $\mathrm{TH}$, we examined the embryonic pigmented wild-type and TH-null fetal tissue for the presence of glyoxylic acid-induced catecholamine histofluorescence in two regions that
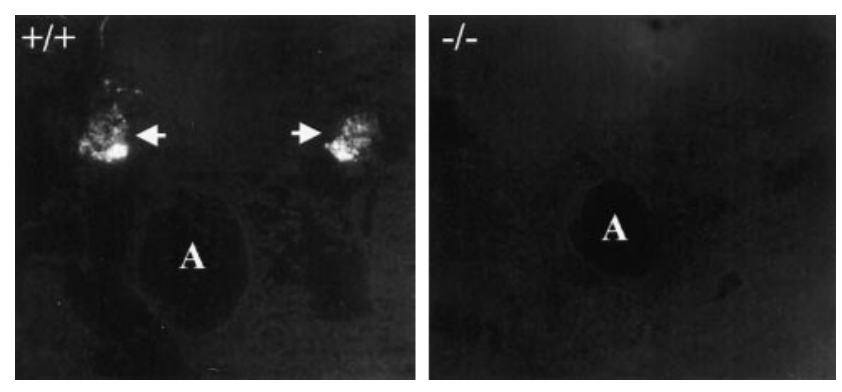

Figure 2. Transverse section through the thoracic region containing the paravertebral sympathetic ganglia. Transverse sections of fetal sympathetic ganglia (arrows) treated with glyoxylic acid revealed catecholamine fluorescence in the wild-type fetus (left), whereas histofluorescence was undetectable in the mutant (right). A, Aorta.

contain TH-positive cells at E13.5. The glyoxylic acid-induced fluorescence method is a sensitive assay for the detection of localized stores of catecholamines, which might not be detectable by quantitative biochemical assays of embryo homogenates. At this developmental stage, catecholamines are present in only a relatively small proportion of normal embryonic tissues. For example, at this stage, sympathetic ganglia have formed, but peripheral tissues are not yet innervated (DeChamplain et al., 1970; Fernholm, 1971). Using this technique, we readily detected catecholamine-containing cells in thoracic sympathetic ganglia of normal littermates (Fig. 2, left). We did not, however, observe any catecholamine histofluorescence in thoracic sympathetic ganglia of mutant fetuses (Fig. 2, right). Similarly, no catecholamine histofluorescence could be detected in the brainstem of the THnull mutant embryos either at the sites of noradrenergic cell bodies in the pons medulla or in the ventral midbrain in which the dopamine-containing neurons reside. In contrast, serotonincontaining cell bodies were visible in the raphe nuclei of both TH-null and wild-type mice (data not shown). Thus, the data show that the mutant $\mathrm{TH}$ does not retain significant catalytic activity.

\section{Rescue of TH-null embryos}

Administration of catecholamine precursor compounds in the drinking water of pregnant dams rescued TH-null embryos in the present study, as was reported previously (Zhou et al., 1995). Two precursor compounds were used for rescue of the pigmented TH-null mutants, both substrates for DDC: L-Dopa and Dops. L-Dopa is converted to dopamine, whereas Dops is converted to norepinephrine. Treatment of pregnant heterozygous females with L-Dopa ( $1 \mathrm{mg} / \mathrm{ml}$ in the drinking water) from E8.5 until birth resulted in the rescue of $90 \%$ of the expected TH-null mice. Treatment with $0.5 \mathrm{mg} / \mathrm{ml}$ Dops rescued $42 \%$ of the expected mutants. Increasing the concentration of Dops to $1 \mathrm{mg} / \mathrm{ml}$ did not enhance survival; only $39 \%$ of the mutants were rescued with this dose. These data indicate that rescue is most successful when all three catecholamines, dopamine, norepinephrine, and epinephrine, are restored by administration of L-Dopa rather than Dops, which is a precursor for norepinephrine and epinephrine but not dopamine. The albino TH-null mice used in this study were rescued with administration of L-Dopa.

Despite the absolute requirement for catecholamines before birth, the pups that lacked $\mathrm{TH}$ and that had been rescued with catecholamine precursors during embryonic development survived for up to 3 weeks after birth without the provision of additional exogenous catecholamine precursors as also observed 
by others (Zhou et al., 1995). Although the TH-null pups appeared to eat normally, they were consistently smaller than their littermates after approximately postnatal day 5 (P5), and did not survive weaning.

\section{Catecholamine histofluorescence in the peripheral and CNS of pigmented postnatal TH-null mice}

Because provision of catecholamine precursors was discontinued at birth and the half-life of catecholamines is $<10 \mathrm{hr}$ in most organs (Iversen and Glowinski, 1966), it was possible to examine the TH-null pups after virtually all the catecholamines produced during the perinatal period would be expected to have been metabolized. In this way, we could ascertain whether catecholamines could be produced by alternative pathway(s). Surprisingly, catecholamine histofluorescence was present in sympathetically innervated peripheral tissues from rescued pigmented TH-null pups at P6 and P15, the two ages examined (Fig. 3). The histofluorescence was present, although the TH-null pups had not received catecholamine precursor supplements since birth. The intense glyoxylic acid-induced histofluorescence characteristic of adrenal chromaffin cells of wild-type mice is shown in Figure $3 a$. The adrenal chromaffin cells of TH-null mice also displayed catecholamine histofluorescence, but the number of fluorescent cells and their fluorescence intensity was reduced compared with the wild-type tissue (Fig. $3 b$ ). In the superior cervical ganglia (SCG) of wild-type pups, cell bodies and processes of principal neurons exhibited bright granular catecholamine histofluorescence (Fig. 3d). In contrast, catecholamine fluorescence was undetectable in the principal neurons of the SCG from TH-null mice. However, the small intensely fluorescent (SIF) cells in the SCG of TH-null mice, like those of wild-type, were brightly fluorescent (Fig. 3e). Although the cell bodies of sympathetic neurons of TH-null mice lacked catecholamine fluorescence, axon terminals in target tissues were fluorescent. Many intensely fluorescent sympathetic fibers were observed in sections of heart ventricles from wild-type mice (Fig. $3 g$ ). Although they were less numerous than in wild-type mice, catecholamine fluorescent fibers were evident in ventricles from TH-null mice (Fig. $3 h$ ). The hairy skin of both wild-type and TH-null P6 pups contained fluorescent sympathetic fibers associated with piloerectors (Fig. $3 j, k)$. Fibers present in the latter exhibited intermediate to weak fluorescence compared with the bright fluorescence of wild-type mice (Fig. 3k). A similar pattern of reduced histofluorescence was observed in TH-null mice compared with wild-type siblings at P15 (data not shown).

To determine whether catecholamines were also present in the CNS of pigmented TH-null mice, the substantia nigra of the ventral midbrain, which is the source of the nigrostriatal tract, and the caudoputamen, its striatal target, were examined. In general, catecholamine histofluorescence was much less prominent in the brains of the pigmented TH-null mutants than in the periphery. Neurons in the substantia nigra, pars compacta of P6 pigmented heterozygous controls are intensely fluorescent because of their high dopamine content (Fig. 4C). In contrast, catecholamine fluorescent neurons were rare in the ventral midbrains of P6 TH-null mice (Fig. 4D). However, the few catecholaminecontaining neurons found in the midbrains of the TH-null pups were brightly fluorescent (Fig. 4D). The patchy dopaminecontaining projections to the striatum that originate with a subset of the neurons in the substantia nigra were intensely fluorescent in the striatum of heterozygous pups (Fig. 4A). In contrast, very little histofluorescence was detected in the striatum of TH-null
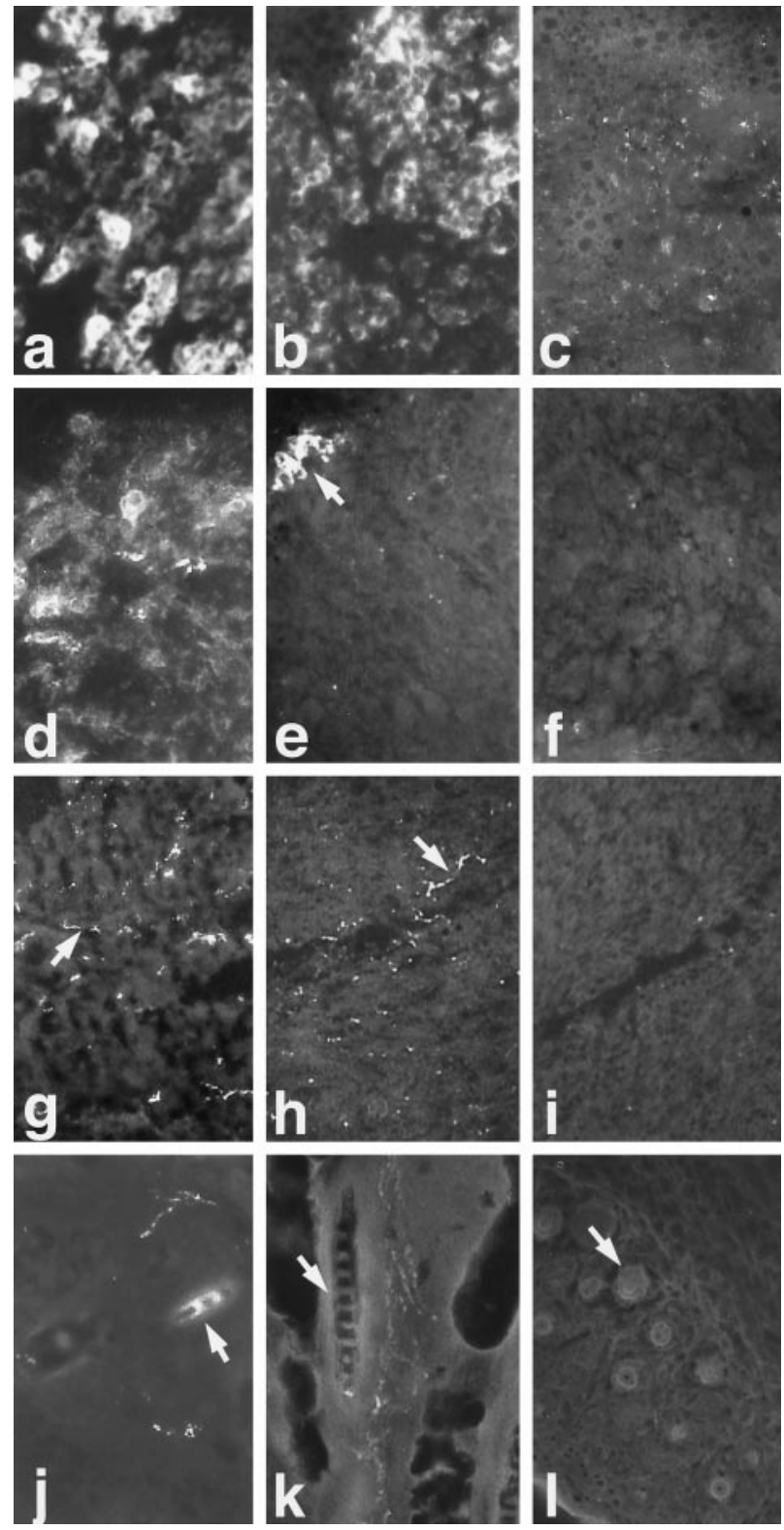

Figure 3. Catecholamine histofluorescence in peripheral tissues of rescued pigmented and albino TH-deficient mice. Adrenal medullae from P6 mice $(a, b, c)$. Adrenal chromaffin cells of pigmented wild-type mice $(a)$ possess intense catecholamine fluorescence. Adrenal chromaffin cells of pigmented TH-null mice $(b)$ contain catecholamines, but the fluorescence is less intense than that of wild-type mice. In contrast, adrenal chromaffin cells of albino TH-null mice $(c)$ are almost devoid of catecholamine histofluorescence. The medullary region with sparse granular fluorescence is distinguishable from the surrounding cortex by the lipid inclusions in the cortical cells. Most sympathetic SCG neurons in pigmented P6 wild-type mice $(d)$ contain bright granular fluorescence. Principal sympathetic neurons of pigmented P6 TH-nulls $(e)$ lacked catecholamine fluorescence; however, fluorescence was seen in SIF cells (arrow). No catecholamine fluorescence could be detected in SCG from TH-nulls that were also tyrosinase-deficient $(f)$. Brightly fluorescent sympathetic fibers were present in the ventricular smooth muscle wall in pigmented P6 wild-type mice $(g)$. A reduced number of brightly fluorescent fibers (arrow) were present in the ventricles of pigmented P6 TH-null mice $(h)$ No catecholamine fluorescent fibers could be detected in the ventricles of albino TH-null mice $(i)$. Brightly fluorescent sympathetic fibers were associated with piloerectors in the hairy skin of pigmented P6 wild-type mice $(j)$. Fibers exhibiting intermediate or weak fluorescence were present in pigmented P6 TH-null mice $(k)$. No catecholamine histofluorescent fibers were detected in the hairy skin of albino TH-null mice $(l)$. 

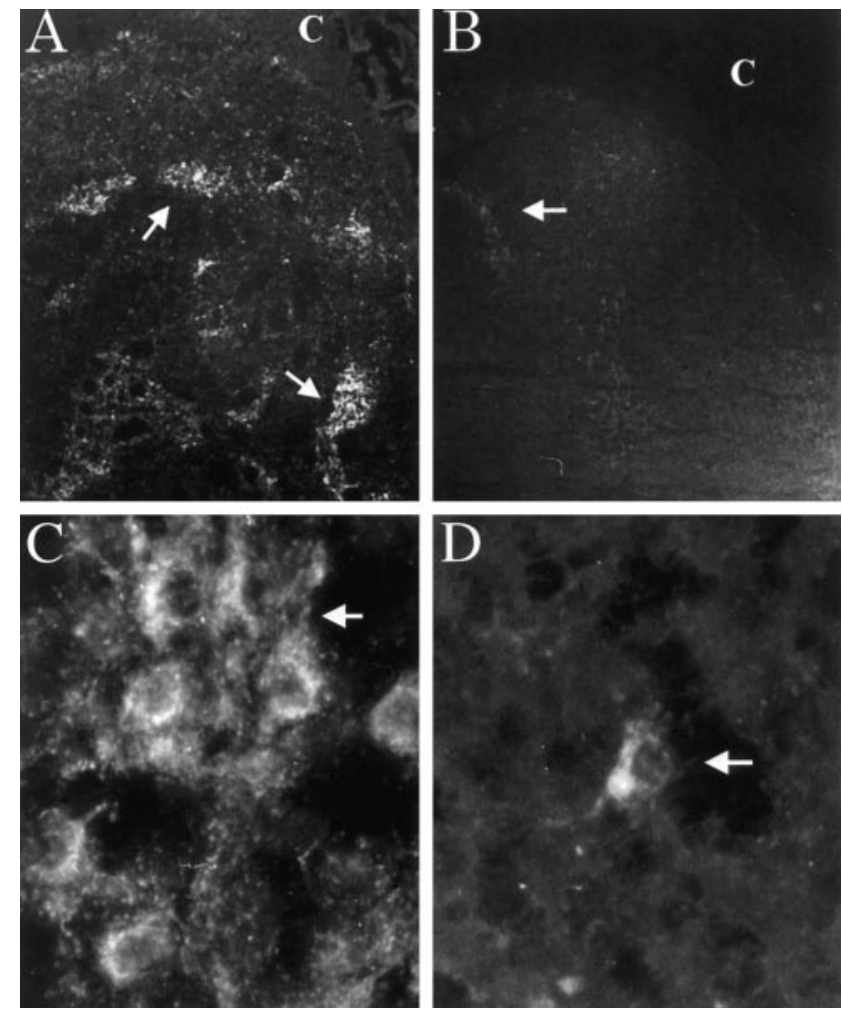

Figure 4. Catecholamine histofluorescence in the striata and ventral midbrains of rescued pigmented TH-null $\mathrm{P} 6$ pups. Coronal sections of the rostral striatum from heterozygous $\mathrm{P} 6$ pups $(A)$ revealed clusters of brightly fluorescent nigral fibers, the dopamine islands (arrows). In contrast, glyoxylic acid treatment of coronal sections of rostral striatum from a pigmented TH-null littermate $(B)$ revealed very few clusters of fluorescent fibers. Those detected were weakly fluorescent (arrow). In heterozygous pups, brightly fluorescent cells in ventral midbrain $(C)$ were abundant in the dopamine-containing substantia nigra, pars compacta. In contrast, the substantia nigra of pigmented TH-null pups $(D)$ contained few catecholamine fluorescent cells. Although such cells were rare, they were brightly fluorescent (arrow). $c$, Cortex.

mice (Fig. 4B), but a few weakly fluorescent patches were visible in some rostral sections of every pigmented TH-null mouse examined.

\section{Lack of glyoxylic acid-induced histofluorescence in albino TH-null pups}

It is unlikely that the catecholamines observed in the postnatal pigmented TH-null mice arose from the catecholamine precursors delivered before but not after birth because the turnover of catecholamines is known to be relatively fast in most tissues, a matter of hours (Iversen and Glowinski, 1966).

One possible source of L-Dopa in the pigmented TH-null pups is tyrosinase; L-Dopa is formed in the melanin pathway through the action of tyrosinase. To determine whether the catecholamines observed in pigmented TH null-mice were synthesized from L-Dopa produced by tyrosinase, catecholamine histofluorescence was examined in double mutants lacking both $\mathrm{TH}$ and tyrosinase. These mice were generated by crossing the $\mathrm{TH}$ targeted allele into ICR mice, a tyrosinase-deficient albino strain. Like the pigmented TH-null mutants, the albino TH-null mutants were rescued in utero by treatment of the pregnant dams with L-Dopa supplied in the drinking water. L-Dopa treatment was discontinued when the pups were born. The distribution and intensity of catecholamine fluorescence in the tissues from P6
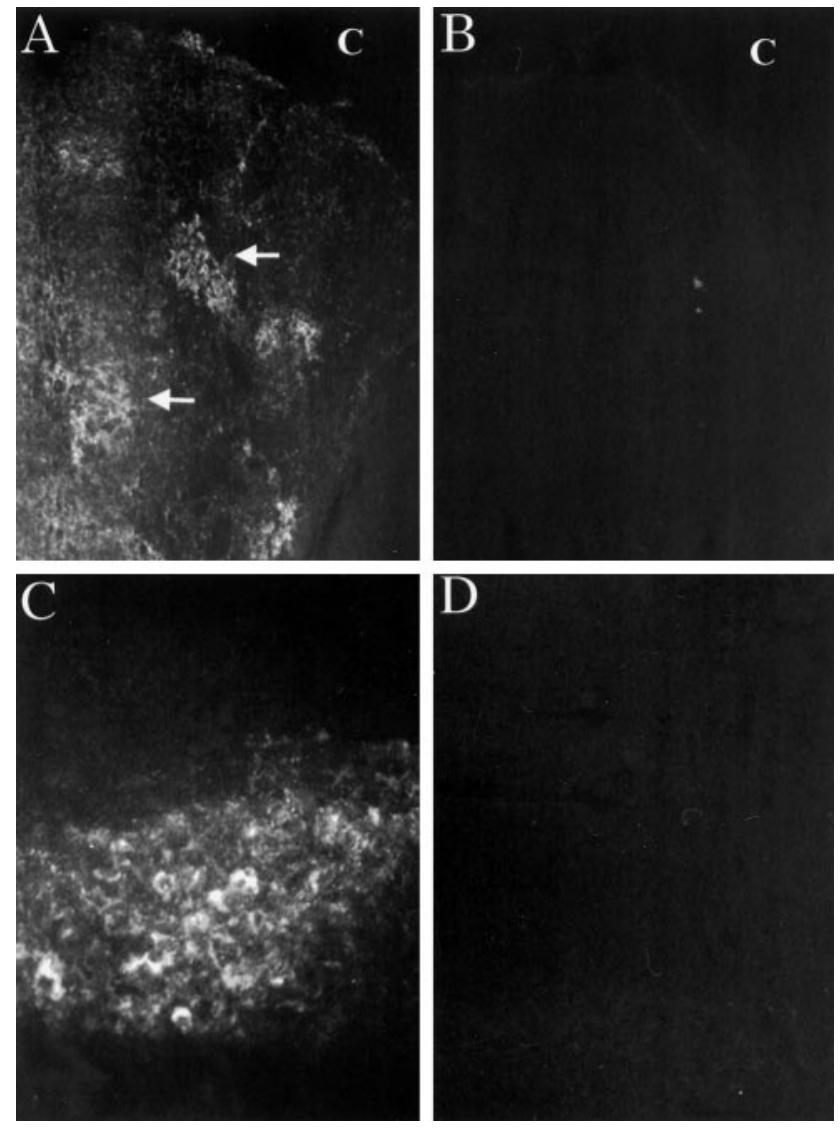

Figure 5. Glyoxylic acid-induced catecholamine fluorescence in the striata and midbrains of albino (tyrosinase-deficient) wild-type and TH-null P6 pups. Striata from wild-type albino mice $(A)$ contain clustered brightly fluorescent fibers, representing dopamine islands (arrows). No catecholamine histofluorescence was seen in the striata of albino TH-deficient mice $(B)$. In the wild-type albino midbrain $(C)$, many fluorescent dopamine cells can be observed. In contrast, no catecholamine histofluorescence could be detected in the midbrains of TH-null mice also deficient in tyrosinase $(D) . c$, Cortex.

albino mice that were wild-type at the TH locus were indistinguishable from those seen in wild-type pigmented pups. In contrast, virtually no catecholamine histofluorescence was seen in the adrenal gland, and none was seen in the SCG, the heart, or hairy skin from P6 albino tyrosinase-deficient pups with disrupted TH alleles (Fig. 3c,f,i,l).

Midbrains and striata from P6 TH and albino tyrosinasedeficient double mutants and their $\mathrm{TH}$ wild-type siblings were also examined for the presence of glyoxylic acid-induced histofluorescence. Whereas TH wild-type tyrosinase-deficient pups had fluorescence patterns and intensity like those of pigmented wild-type and heterozygous pups, the midbrains and striata from tyrosinasedeficient TH-nulls were devoid of catecholamine fluorescence (Fig. $5 B, D)$. These results, together with those from peripheral tissues, provide compelling evidence that tyrosinase mediates catecholamine production in the absence of functional $\mathrm{TH}$.

\section{Endogenous catecholamine content of pigmented TH- null mice and albino TH-null mice compared with heterozygotes and wild-type controls}

To confirm that the glyoxylic acid-induced histofluorescence observed in pigmented TH-null postnatal mice reflected the presence of catecholamine and to obtain quantitative measures of 


\begin{tabular}{|c|c|c|c|c|c|c|}
\hline & \multirow{2}{*}{$\frac{\text { Heart }}{\mathrm{NE}(\mathrm{pg} / \mathrm{mg})}$} & \multirow{2}{*}{$\frac{\text { Skin }}{\mathrm{NE}(\mathrm{pg} / \mathrm{mg})}$} & \multicolumn{2}{|l|}{ Adrenal } & \multicolumn{2}{|l|}{ Brain } \\
\hline & & & NE (ng/pair) & E (ng/pair) & $\mathrm{NE}(\mathrm{pg} / \mathrm{mg})$ & $\mathrm{DA}(\mathrm{pg} / \mathrm{mg})$ \\
\hline -/- Albino & $6.1 \pm 1.6$ & $0.1 \pm 0.0$ & $0.2 \pm 0.0$ & $0.3 \pm 0.0$ & $3.0 \pm 1.3$ & $1.4 \pm 0.6$ \\
\hline -/- Pigmented & $63 \pm 16^{\dagger}$ & $7 \pm 2^{\dagger}$ & $7 \pm 1^{\dagger}$ & $9 \pm 1^{\dagger}$ & $22 \pm 4^{\dagger}$ & $11 \pm 2^{\dagger}$ \\
\hline$+/-$ Albino & $372 \pm 45^{*}$ & $28 \pm 3^{*}$ & $321 \pm 38^{*}$ & $513 \pm 77^{*}$ & $214 \pm 11^{*}$ & $378 \pm 15^{*}$ \\
\hline$+/-$ Pigmented & $277 \pm 40^{*}$ & $24 \pm 6^{*}$ & $220 \pm 35^{*}$ & $364 \pm 54^{*}$ & $215 \pm 8^{*}$ & $365 \pm 16^{*}$ \\
\hline$+/+$ Albino & $424 \pm 40^{*}$ & $50 \pm 8^{* \S}$ & $417 \pm 96^{*}$ & $622 \pm 141^{*}$ & $239 \pm 11^{*}$ & $461 \pm 29^{* \S}$ \\
\hline$+/+$ Pigmented & $376 \pm 29 * \S$ & $32 \pm 5^{*}$ & $227 \pm 30^{*}$ & $440 \pm 71^{*}$ & $277 \pm 19^{* \$}$ & $483 \pm 16^{* \S}$ \\
\hline
\end{tabular}

Values are mean \pm SEM of values from 6 to 10 mice except the $-/-$ albino skin value, which is from 4 mice.

Wild-type $=+/+$; heterozygote $=+/-$; TH-null $=-/-$.

* Significant difference $(p<0.05)$ between wild-type or heterozygote mice compared with corresponding TH-null mice (Sheffe's $F$ test).

† Significant difference $(p<0.05)$ between pigmented TH-null mice and the corresponding albino TH-null mice (Sheffe's $F$ test).

$\S$ Significant difference $(p<0.05)$ between wild-type mice compared with corresponding heterozygote mice (Sheffe's $F$ test).

Content of Catecholamines in Pigmented and Albino TH-nulls

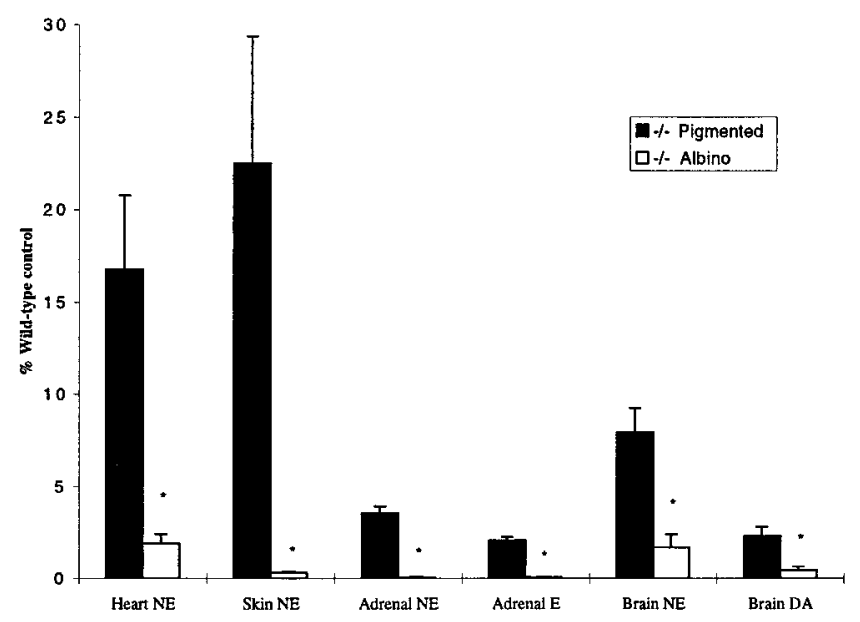

Figure 6. Catecholamines were extracted from tissues harvested from TH-null and wild-type pups that were 14- and 15-d-old. Both the albino and pigmented mice were treated until birth with L-Dopa supplied to pregnant dams. Values represent the mean \pm SEM of six or seven samples, except skin of the albino TH-null, which is the mean of three samples. Values are calculated as percent of the appropriate (albino or pigmented) wild-type value. ${ }^{*} p<0.005$ indicates a significant difference from values for pigmented mice by Student's $t$ test.

catecholamines in pigmented and albino TH-null animals, catecholamines were measured in peripheral tissues and brain of P15 mice of all genotypes. Although the reduction in catecholamines caused by the lack of TH and/or tyrosinase varied among tissues, as described below and in Table 1 and Figure 6, we found that catecholamines were reduced by the presence of one or two mutant $\mathrm{TH}$ alleles and that these reductions were much greater in the absence of tyrosinase. Together, our results indicate that, in the absence of $\mathrm{TH}$, tyrosinase plays an important role in supplying Dopa for catecholamine synthesis.

Whereas there were no significant differences in catecholamine content in any tissues between pigmented and albino wild-type pups (Table 1), the presence of one mutant TH allele compromised the catecholamine content of tissues (Table 1). The catecholamine content of every tissue from the heterozygous pups was lower than that of the corresponding tissue from wild-type pups; several of these differences were significant. The significant differences found were between the pigmented wild-type heart and its heterozygous counterpart (norepinephrine in the heterozygote was $74 \%$ that of wild-type), between the wild-type albino skin and the heterozygous albino skin (norepinephrine was 56\% of that in the wild-type), and in brain for both norepinephrine (78\% of wild-type) and dopamine (76\% of wild-type) in the pigmented mice and in brain dopamine ( $82 \%$ of wild-type) in the albino mice.

Although the catecholamine content of tissues from P15 pigmented TH-null mice were dramatically and significantly reduced, every tissue assayed did, in fact, contain catecholamines (Table 1). Catecholamine content in pigmented TH-null pups was $22 \%$ or less of wild type, consistent with the major role of $\mathrm{TH}$ in catecholamine synthesis (Table 1, Fig. 6). In peripheral tissues, the norepinephrine content of the TH-null pups ranged from $3 \%$ of wild-type in the adrenal medulla to $22 \%$ in hairy skin. In brain, dopamine content was $\sim 2 \%$ of wild-type values. Norepinephrine content, however, was $8 \%$ of wild-type.

When we compared catecholamine content in tissues from albino TH-null mice with those in albino wild-type mice, we found a greater reduction in the TH-null albino pups than was observed in the pigmented TH-null mice (Table 1, Fig. 6). The catecholamine content of the albino TH-null mice was $<18 \%$ those of the pigmented TH-null pups (Table 1, Fig. 6). Norepinephrine content in the hairy skin of pigmented TH-null mice was $22 \%$ of wild-type, whereas it was $<0.5 \%$ of wild-type values in albino TH-null pups. In the hearts from TH-null pigmented mice, norepinephrine was $17 \%$ of wild-type, whereas in TH-null albino hearts, it was $2 \%$, more than sixfold lower. Whereas in the adrenal glands from the TH-null pigmented mice norepinephrine and epinephrine were $3 \%$ and $2 \%$, respectively, that of pigmented wild-type mice, $<0.06 \%$ of wild-type albino levels for norepinephrine and epinephrine were found in the adrenals of albino TH-null mice. In brain, catecholamines in the albino TH-nulls were $18 \%$ of those in the pigmented TH-null mice (Table 1, Fig. 6).

\section{DISCUSSION}

Examination of the pigmented TH-null postnatal mice uncovered an alternate pathway for synthesis of catecholamines that was detected histologically by glyoxylic acid-induced histofluorescence and biochemically after HPLC separation of extracted catecholamines. Based on a comparison of our TH-null mutation on a pigmented and an albino background, the data indicate that 
tyrosinase can supply L-Dopa for catecholamine synthesis to catecholaminergic neurons and chromaffin cells. Albino mice that lack both $\mathrm{TH}$ and tyrosinase also lack glyoxylic acid-induced histofluorescence in the adrenal medulla, in sympathetically innervated tissues, and in brain. Consistent with the role of tyrosinase in catecholamine synthesis, the tissues from the double mutants contained fivefold less catecholamine than did pigmented TH-null mice.

An alternative explanation for the presence of catecholamines in the pigmented TH-null mice is that they are derived from the exogenous catecholamine precursors that were provided to the embryos from E8.5 until birth. However, the catecholamines that rescued the mutant embryos are unlikely to be the source of the catecholamines found postnatally in the pigmented TH-null mutants. Because catecholamines have a relatively short half life, (Iversen, 1967), those formed in utero should be undetectable several days after birth: at least 14 half-lives had passed before the P6 tissues were analyzed. In addition, tissues from 15-d-old pigmented TH-null pups were examined for catecholamine histofluorescence, and the fluorescence was no less prominent at $15 \mathrm{~d}$ than at $6 \mathrm{~d}$. Moreover, the quantitative catecholamine measurements were from P14 and P15 tissues. The most compelling argument, however, that the catecholamines do not come from a persistent perinatal store is that, although both pigmented and albino mice received L-Dopa during gestation, only the pigmented TH-nulls contained sufficient catecholamine to be detected by glyoxylic acid-induced fluorescence during postnatal life.

The source(s) of the tyrosinase that contributes to catecholamine synthesis in pigmented TH-null mice is unknown. Several different classes of melanin-producing cells express high levels of tyrosinase. The best known are melanocytes, derived from the neural crest and present in skin, hair follicles, and the choroid of the eye. In addition, the retinal pigment epithelium, which is derived from the optic cup, contains high levels of tyrosinase. Several studies raise the possibility that tyrosinase is also expressed in the CNS. Tyrosinase mRNA is found in the human substantia nigra (Xu et al., 1997), and tyrosinase-like activity has been reported there (Miranda et al., 1984). Tyrosinase transcripts and protein are also present in newborn and adult mouse brains (Tief et al., 1996a), and mice with a tyrosinase promoter-lacZ fusion gene express the transgene throughout the neural tube (Tief et al., 1996b). However, tyrosinase activity has not been detected in the mouse brain (Tief et al., 1996a). Therefore, it seems more likely that the substrates for catecholamine synthesis in the brains of pigmented TH-nulls come from the circulation than from a local source. It is clear that melanocytes represent one source for L-Dopa in the periphery, but it is possible that other sources also exist.

A likely scenario is that L-Dopa generated by the melanin pathway in melanocytes becomes available to and is accumulated by catecholaminergic cells in which it is converted to dopamine, norepinephrine, and epinephrine. Additionally or alternatively, melanocyte-derived L-Dopa could be converted to dopamine extraneuronally by DDC and the resulting dopamine subsequently accumulated by catecholaminergic cells. Quantification of serum Dopa in albino, black, and red guinea pigs provide evidence first that significant levels of Dopa are present in serum and second that Dopa is more abundant in the serum of pigmented animals. Whereas serum Dopa was 0.4 and $0.7 \mathrm{ng} / \mathrm{ml}$ in white and red guinea pigs, it was $1.9 \mathrm{ng} / \mathrm{ml}$ in black animals (Hansson et al., 1980). In preliminary studies, we have found that the plasma levels of Dopa are more than fivefold higher in pigmented 15-dold wild-type pups than in albino wild-type pups of the same age. Furthermore, Dopa is present in serum obtained from TH-null pups. Thus, plasma Dopa could provide precursor for catecholamine synthesis in the periphery and in brain, and it is likely that more Dopa is available for catecholamine synthesis in pigmented mice than in albinos.

Although the content of catecholamines was extremely low in tissues from our TH-null albino pups, catecholamines were not completely absent, indicating that yet another pathway may be present. The nature of the residual catecholamines is unclear. Whether the catecholamines present in, for example, the hearts and brains of the TH-null albino pups are extraneuronal or are concentrated in neurons is unknown. Because they exist at such low concentrations, the residual catecholamines are undetectable with glyoxylic acid-induced histofluorescence, and hence their location is difficult to assess. The tissues from postnatal heterozygotes on both the albino and the pigmented backgrounds had reductions in catecholamines. Given the many mechanisms by which tyrosine hydroxylase activity is regulated, it is interesting to note that a single wild-type TH allele is insufficient to completely compensate for the lack of the second normal allele, although it comes close.

The pattern of catecholamine histofluorescence observed in the pigmented TH-null animals differs in several ways from that observed in wild-type animals. The presence of catecholamine histofluorescence in pigmented TH-null mice is correlated with the density of vesicular stores in the individual peripheral tissues. Catecholamine histofluorescence is present where storage vesicles are numerous: in the adrenal medulla, in all sympathetically innervated tissues examined, and in the SIF cells of the SCG. Catecholamine fluorescence was not detected in the cell bodies of principal neurons of the SCG from the mutants but was present in sympathetic axons in which catecholamine storage vesicles are numerous. Although sympathetic terminals visualized by histofluorescence were less numerous in the pigmented TH-null mice than in either heterozygous or wild-type mice, those terminals visible in the TH-null mice were brightly fluorescent. We do not know whether sympathetic innervation is reduced in the mutant or whether only a subset of the terminals present contain catecholamines.

Catecholamine histofluorescence was also found in the brains of the TH-null pups but was far less prominent there than in peripheral tissues. Few fluorescent neurons were found in the substantia nigra, pars compacta, a cell group that normally contains thousands of dopamine-producing neurons. The paucity of fluorescent cells in the mutant is not caused by absence of cells because neurons are detected in the substantia nigra with antibodies to DDC (our unpublished results; Kobayashi et al., 1995; Zhou and Palmiter, 1995. The rare fluorescent neurons seen in the mutant's substantia nigra, pars compacta were intensely bright, indicating that the catecholamine present in the nigrostriatal system is unevenly distributed. Where detected, the dim patchy fluorescence in the rostral striatum of pigmented TH-null mice displayed the distribution characteristic of the clustered early arriving afferents from the substantia nigra that are normally present in young pups.

Together, the data indicate that neurons in the rescued pigmented TH-null postnatal mice can capture Dopa or dopamine synthesized because of the presence of tyrosinase and use them as substrates for catecholamine synthesis. However, it is unclear whether catecholamines synthesized via tyrosinase play a physi- 
ological role in the pigmented TH-null mice. Certainly during prenatal development, tyrosinase does not supply sufficient Dopa to sustain survival or to produce sufficient catecholamine to be detectable by histofluorescence. This is likely to be a result of insufficient catecholamine production rather than to deficits in Dopa accumulation into catecholamine-producing cells, because rescue is accomplished by supplying circulating Dopa through the dam. Tyrosinase activity may appear too late during embryonic development to rescue the TH-null embryos. Because tyrosinase is not detected in melanocytes until E17 (Beermann et al., 1992), catecholamine content is likely to be below the minimum required for survival during the E11-E14 window when most THnull mice die in utero, although little catecholamine appears to be required for survival. For example, only $10 \%$ of the normal norepinephrine content was sufficient for almost full rescue of $\mathrm{D} \beta \mathrm{H}$-deficient embryos rescued with Dops in utero (Thomas et al., 1995), suggesting that tyrosinase fails to restore even this low level of catecholamines in our pigmented TH-null embryos. Consistent with this interpretation, Kobayashi et al. (1995) failed to detect norepinephrine after HPLC separation of catecholamines from pigmented E12.5 TH-null embryos.

In our view, the presence of surprisingly high concentrations of catecholamines in sympathetically innervated tissues and even in the brains of postnatal pigmented mice without $\mathrm{TH}$ is probably not attributable to a compensatory mechanism in which tyrosinase has been upregulated as a consequence of the elimination of TH. Examination of the several strains of TH-null mice generated establish that $\mathrm{TH}$ is an essential gene for embryonic development; there is no substitute for TH. Rather, our data demonstrating the presence of catecholamines in the pigmented TH-null pups provide evidence of the unmasking of a source of L-Dopa for catecholamine synthesis that may always be present in pigmented animals after birth, as has long been suspected. It remains to be determined whether tyrosinase, although it is an inefficient source of L-Dopa for catecholamine synthesis, may be able to partially restore catecholamine function in pigmented postnatal TH-null mice. Finally, it will be of interest to learn whether L-Dopa derived from tyrosinase supports catecholaminergic functions in normal animals.

\section{REFERENCES}

Beermann F, Schmid E, Ganss R, Schutz G, Ruppert S (1992) Molecular characterization of the mouse tyrosinase gene: pigment cell-specific expression in transgenic mice. Pigm Cell Res 5:295-299.

Blaschko H (1939) The specific action of L-dopa decarboxylase. J Physiol (Lond) 96:50-51.
De la Torre J (1980) An improved approach to histofluorescence using the SPG method for tissue monoamines. J Neurosci Methods 3:1-5.

DeChamplain J, Malmfors T, Olson L, Sachs C (1970) Ontogenesis of peripheral adrenergic neurons in the rat: pre- and postnatal observations. Acta Physiol Scand 80:276-288.

Eisenhofer G, Goldstein D, Stull R, Keiser H, Sunderland T, Murphy D, Kopin I (1986) Simultaneous liquid chromatographic determination of 3,4-dihydroxyphenylglycol, catecholamines and 3,4-dihydroxyphenylalanine in plasma, and their responses to monoamine oxidase inhibition. Clin Chem 32:2030-2033.

Fernholm M (1971) On the development of the sympathetic chain and adrenal medulla in the mouse. Z Anat Entwicklungsescg 133:305-317.

Hansson C, Agrup G, Rorsman H, Rosengren E (1980) Dopa and 5-Scysteinyldopa in the serum of albino, black, and red guinea pigs. Acta Dermato-Venereol 60:155-156.

Ichikawa S, Sasaoka T, Nagatsu T (1991) Primary structure of mouse tyrosine hydroxylase from its cDNA. Biochem Biophys Res Commun 176:1610-1616.

Iversen L (1967) The uptake and storage of noradrenaline in sympathetic nerves, pp. 51-82. Cambridge, MA: Cambridge UP.

Iversen L, Glowinski J (1966) Regional studies of catecholamines in the rat brain. II. Rate of turnover of catecholamines in various brain regions. J Neurochem 13:671-682.

Kobayashi K, Morita S, Sawada H, Mizuguchi T, Yamada K, Nagatsu I, Hata T, Watanabe Y, Fujita K, Nagatsu T (1995) Targeted disruption of the tyrosine hydroxylase locus results in severe catecholamine depletion and perinatal lethality in mice. J Biol Chem 270:27235-27243.

Miranda M, Botti D, Bonfigli A, Ventura T, Arcadi A (1984) Tyrosinase-like activity in normal human substantia nigra. Gen Pharmacol 15:541-544.

Nagatsu T, Levitt M, Udenfriend S (1964) Tyrosine hydroxylase: the initial step in norepinephrine biosynthesis. J Biol Chem 239:2910-2917.

Ribeiro P, Wang Y, Citron B, Kaufman S (1993) Deletion mutagenesis of rat PC12 tyrosine hydroxylase regulatory and catalytic domains. J Mol Neurosci 4:125-139.

Sanchez-Ferrer A, Rodriguez-Lopez J, Garcia-Canovas F, GarciaCarmona F (1995) Tyrosinase: a comprehensive review of its mechanism. Biochim Biophys Acta [Review] 1247:1-11.

Thomas S, Matsumoto A, Palmiter R (1995) Noradrenaline is essential for mouse fetal development. Nature 374:643-646.

Tief K, Hahne M, Schmidt A, Beermann F (1996a) Tyrosinase, the key enzyme in melanin synthesis, is expressed in murine brain. Eur J Biochem 241:12-16.

Tief K, Schmidt A, Aguzzi A, Beermann F (1996b) Tyrosinase is a new marker for cell populations in the mouse neural tube. Dev Dyn 205:445-456.

Xu Y, Stokes A, Freeman W, Kumer S, Vogt B, Vrana K (1997) Tyrosinase mRNA is expressed in human substantia nigra. Brain Res Mol Brain Res 45:159-162.

Zhou Q, Palmiter R (1995) Dopamine-deficient mice are severely hypoactive, adipsic, and aphagic. Cell 83:1197-1209.

Zhou Q, Quaife C, Palmiter R (1995) Targeted disruption of the tyrosine hydroxylase gene reveals that catecholamines are required for mouse fetal development. Nature 374:640-643. 